\title{
Transarterial chemoembolization combined with apatinib for treatment of advanced hepatocellular carcinoma: analysis of survival and prognostic factors
}

\section{Ziyi Liu}

Wuhan Union Hospital

Xuefeng Kan

Wuhan Union Hospital

Lijie Zhang

Wuhan Union Hospital

Joyman Makamure

Wuhan Union Hospital

Qing Li

Wuhan Union Hospital

Dan Zhao

Wuhan Union Hospital

Guofeng Zhou

Wuhan Union Hospital

\section{Gansheng Feng}

Wuhan Union Hospital

Chuansheng Zheng

Wuhan Union Hospital

Bin Liang ( $\boldsymbol{\sim}$ bliang@hust.edu.cn )

Wuhan Union Hospital https://orcid.org/0000-0002-6130-8629

Research article

Keywords: Chemoembolization, therapeutic, Apatinib, Survival, Factor analysis, statistical

Posted Date: January 21st, 2020

DOl: https://doi.org/10.21203/rs.2.21435/v1

License: (c) (1) This work is licensed under a Creative Commons Attribution 4.0 International License.

Read Full License 
Page 2/15 


\section{Abstract}

Backgroud: To evaluate the overall survival (OS) after transarterial chemoembolization (TACE) combined with apatinib in patients with advanced hepatocellular carcinoma (HCC), and to identify the factors affecting patient survival.

Methods: Fifty-one patients with HCC who received TACE combined with apatinib in our hospital from June 2015 to May 2017 were enrolled and followed up until May 2019. The OS and progression-free survival (PFS) were calculated using the Kaplan-Meier method. Log-rank test and Cox regression model were used to determine the factors affecting the patients' OS.

Results: The median OS and PFS of the patients were 15 months and 10 months, respectively. The 1-, 2-, and 3-year survival rates were $62.7 \%, 23.5 \%$, and $1.8 \%$, respectively. Univariate survival analysis showed that patients with Child-Pugh A $(P=0.008)$, severe reduction rate of proper hepatic artery $(P=0.013)$, hand-foot syndrome $(P=0.006)$, secondary hypertension $(P=0.042), \mathrm{AFP}<400 \mathrm{ug} / \mathrm{L}(P=0.076)$, and without ascites $(P=0.013)$ had a better OS. Multivariate analysis showed that hand-foot syndrome $(P=$ $0.004)$, secondary hypertension $(P=0.034)$, and reduction rate of proper hepatic artery $(P=0.028)$ were independent predictors of better OS.

Conclusions: TACE combined with apatinib is a promising treatment for advanced HCC. Hand-foot syndrome, secondary hypertension, and the reduction rate of proper hepatic artery were associated with a better OS.

\section{Background}

Hepatocellular carcinoma (HCC) is the sixth most common malignancy and the third leading cause of cancer death globally, and more than $50 \%$ of HCC cases occur in China [1, 2]. Current treatments for HCC include ablation, resection, liver transplantation, transarterial chemoembolization (TACE), systemic therapies, and optimal supportive care [1, 2]. Unfortunately, a considerable number of Chinese patients are in the advanced stage when diagnosed with HCC, resulting in a poor prognosis. Sorafenib, an oral multikinase inhibitor that has antiproliferative and antiangiogenic effects, is the standard systemic therapy for advanced HCC, but its long-term efficacy remains unsatisfactory.

Although transcatheter chemoembolization (TACE) is recommended for intermediate-stage HCC, in real life the procedure has been used to treat advanced-stage liver cancer $[1,3]$. The rationale for TACE stems from the observation that liver cancer receives most of its blood supply from the hepatic artery, whereas normal liver tissue receives most of its blood supply from the portal vein. TACE, which consists of the intra-arterial infusion of a chemotherapeutic agent followed by embolization of the tumor-feeding arteries, will achieve a substantial cytotoxic and hypoxic/ischemic tumor necrosis [4]. However, the local hypoxia after TACE can activate hypoxia-inducible factor-1 (HIF-1) and up-regulate the level of vascular endothelial growth factor (VEGF), thereby promoting tumor angiogenesis and recurrence [5]. 
The combination of TACE and a systemic therapy that targets angiogenesis may constitute an effective strategy to improve outcomes. Although recent studies have shown that sorafenib combined with TACE failed to improve survival in patients with advanced HCC compared with sorafenib alone [6], we believe that other molecular targeted therapies may prove to be effective, when combined with TACE. Apatinib is another small-molecule tyrosine kinase inhibitor that exhibits higher selective inhibition of VEGF receptor2 than sorafenib, which can block the migration and proliferation of vascular endothelial cells, reduce tumor microvessel density, and inhibit tumor growth [7]. Recent studies have found that adjuvant apatinib can enhance the effectiveness of TACE for advanced HCC [8-12]. This study was designed to evaluate the long-term outcomes of TACE in combination with apatinib for advanced HCC and its predictors.

\section{Methods}

\section{Selection of patients}

A retrospective analysis was conducted of all HCC patients who received TACE combined with apatinib at Union Hospital, Tongji Medical College, Huazhong University of Science and Technology from June 2015 to May 2017. The criteria for patient selection included patients aged $>18$ years with HCC, multinodular or unresectable tumors with macrovascular invasion and/or extrahepatic spread, Eastern Cooperative Oncology Group performance status of $0-2$, liver function Child-Pugh Class A and B, no previous chemotherapy, ablation, radiotherapy or systemic therapy. Patients were excluded if they had diffuse HCC or liver tumor burden $>50 \%$ of total liver volume, complete obstruction of the main portal vein, advanced liver disease (bilirubin $>3 \mathrm{mg} / \mathrm{dl}$, AST or ALT > 5 times normal upper limit or $>250 \mathrm{U} / \mathrm{l}$, refractory ascites, uncontrollable hepatic encephalopathy or hepatorenal syndrome), severe dysfunction of kidney, heart, lung or coagulation. Patients were also excluded if they had discontinued apatinib $>1$ month or were lost to follow-up.

This study was approved by the Ethics Committee of the Wuhan Union Hospital, Tongji Medical College, Huazhong university of Science and Technology. Written informed consent was obtained from all patients.

\section{Combination of TACE and apatinib}

All patients received TACE in combination with apatinib. The TACE procedure was performed under fluoroscopic guidance. Seldinger technique was used to puncture the femoral artery and a vascular sheath was placed. A 5F Yashiro or RH catheter was then used to catheterize the celiac trunk and superior mesenteric artery. Selective arteriography was performed to detect hypervascular masses. A 2.7F Terumo Progreat microcatheter was introduced coaxially through the $5 \mathrm{~F}$ catheter into the branches that supply the tumor. A mixture of single (epirubicin 40-60 mg) or multiple chemotherapeutic agents (epirubicin 40$60 \mathrm{mg}$, oxaliplatin $50 \mathrm{mg}$ and raltitrexed $2-4 \mathrm{mg}$ ) with $5-20 \mathrm{ml}$ of lipiodol was delivered and additional gelatin sponges $350-560 \mu \mathrm{m}$ in size were injected to block the tumor-feeding arteries. The embolization endpoint was complete stasis or near stasis of the blood flow. In cases of bilobar or massive lesions, at least two TACE procedures were performed. 
The apatinib was initiated at an oral dose of $500 \mathrm{mg} / \mathrm{d} 3$ days after TACE. The daily apatinib dose was decreased to $250 \mathrm{mg}$ if patients developed drug-related grade 3 or 4 nonhematologic or hematologic toxicities. If the $250 \mathrm{mg}$ daily dose was not tolerated yet, the apatinib treatment was discontinued. For patients who required multiple TACE procedures, the apatinib was withdrawn 2 days before TACE and resumed 3 days after TACE.

\section{Follow-Up and survival assessment}

Patients were followed up at 1 month after TACE, and then every 2-3 months thereafter. CT scans (noncontrast, arterial and portal venous phases) and laboratory data were used during these visits for surveillance. Evidence of progressive disease followed the modified Response Evaluation Criteria in Solid Tumors. The endpoints of follow-up were death, loss to follow-up or the end of the study (May 2019).

The primary endpoint of this study was overall survival (OS), defined as the time from initiation of the combination treatment to death. Patients who remained alive at the end of follow-up were censored. The secondary endpoint was progression-free survival (PFS), defined as the time from the treatment initiation to either radiological progression or death. Adverse events were monitored and graded according to the National Cancer Institute Common Terminology Criteria for Adverse Events version 4.0.

To identify factors that might predict survival, the following variables were analyzed: hepatitis $B$ virus (HBV), Eastern Cooperative Oncology Group (ECOG) score, Child-Pugh grade, alpha fetoprotein (AFP), number of lesions, size of dominant lesion, macrovascular invasion, extrahepatic spread, ascites, number of TACE procedures, changes in the proper hepatic artery, hand-foot syndrome, secondary hypertension, diarrhea and proteinuria (Table 1). The change in the proper hepatic artery was measured as a percentage of the difference in the vessel diameter between the baseline and the first follow-up DSA images (1-3 month) to the baseline vessel diameter. The median change in the proper hepatic artery of 51 patients was used as the cut-off value, which converted the degree of the vessel change into mild ( $\leq$ median) and severe (> median).

\section{Statistical analysis}

Survival times and survival rates were calculated by using the Kaplan-Meier method. Paired $t$ test was used to compare the difference in the common hepatic artery diameter before and after combination treatment. For OS, the log-rank test and life-table method were used for the univariate analysis. Any predictors that were significant at $P<0.1$ in the univariate analysis were candidates for entry into a Cox regression model for the multivariate analysis. Statistical analysis was performed by using SPSS 17.0 (SPSS Inc., Chicago, III). A $P<0.05$ was considered to indicate a significant difference.

\section{Results}

\section{Patient characteristics}


A total of 98 HCC patients received TACE combined with apatinib in our hospital between June 2015 and May 2017. Eighteen patients were excluded due to apatinib discontinuation, and 29 patients were lost to follow-up. Finally, 51 patients were included in this study (Fig. 1). The overall patient characteristics are shown in Table 1. This cohort consisted of 44 males and 7 females. The mean age was $47.9 \pm 10.1$ years (mean \pm standard deviation). Patients presented with an average of $2.8 \pm 1.8$ lesions (range, 1-8 lesions). The largest tumor size measured $9.3 \pm 5.0 \mathrm{~cm}$ (range, $1.2-19.1 \mathrm{~cm})$.

\section{Treatment process}

Of the 51 patients, 31 had 1 TACE session, 9 had 2 sessions, and 11 had 3 or more sessions before inclusion. After inclusion, 11 patients received 1 TACE session, 10 received 2 sessions, and 30 received 3 or more sessions. The median number of TACE treatments per patient was $3.6 \pm 2.8$ (range, 1-14).

As for the administration of apatinib, 500 mg per day was given in 45 patients, whereas the dose was reduced to $250 \mathrm{mg}$ per day in the other 6 patients due to the development of grade 3 or 4 adverse reactions. The most common grade 3 or 4 adverse reactions occurred in $9.8 \%$ of patients (5/51 patients), which included hand-foot syndrome, secondary hypertension, diarrhea and proteinuria during the entire period of combination treatment (Table 1).

\section{Survival}

The median follow-up time was 15 months (range, 3-38 months). Forty-four (86.3\%) of the 51 patients died and 7 (13.7\%) remained alive during the follow-up period (June 2015 to May 2019). The median OS was 15 months (range, 3-38 months) (Fig. 2a), and the median PFS was 10 months (range, 1-24 months) (Fig. 2b). The cumulative OS rates at 1, 2, and 3 years were $62.7 \%, 23.5 \%$, and $1.8 \%$, respectively.

At univariate analysis, patients with Child-Pugh grade A had significantly longer median OS compared with Child-Pugh grade B patients (17 months vs.10 months, $P=0.008$ ) (Fig. 3a). Patients without ascites had significantly longer median OS compared with those with ascites $(17$ months vs. 9 months, $P=$ 0.013) (Fig. 3b). patients with AFP< $400 \mathrm{ug} / \mathrm{L}$ had significantly longer median OS compared with $A F P \geq$ $400 \mathrm{ug} / \mathrm{L}$ patients (19 months vs.12 months, $P=0.076$ ) (Fig. 3c). The median OS of patients without hand-foot syndrome was 10 months, whereas the median os of patients with grade 1, 2 and 3 hand-foot syndrome was 14, 21 and 17 months, respectively. Patients with grade 2 hand-foot syndrome had significantly longer median OS compared with patients without hand-foot syndrome (21 months vs. 10 months, $P=0.003$ ) (Fig. 3d). The median OS of patients without secondary hypertension was 15 months, whereas that of patients with grade 1, 2 and 3 secondary hypertension was 18, 15.5 and 28 months, respectively. Patients with grade 3 hypertension had significantly longer median OS compared with nonhypertensive patients (28 months vs. 15 months, $P=0.032$ ) (Fig. 3e). The diameter of the proper hepatic artery before and after combination therapy was $3.79 \pm 1.81 \mathrm{~mm}$ and $3.40 \pm 1.66 \mathrm{~mm}$, respectively, with a statistically significant difference $(P<0.001)$. The median reduction rate of the proper hepatic artery after treatment was $7.7 \%$. Patients with mildly reduced diameter of the proper hepatic artery had longer median 
OS than those with severely reduced vessel diameters (23 months vs. 12 months, $P=0.013$ ) (Fig. $3 f$ ) (Table 1).

At multivariate analysis, the hand-foot syndrome $(P=0.004, \mathrm{HR}=0.554)$, secondary hypertension $(P=$ $0.034, \mathrm{HR}=0.647)$, and the reduction rate of the proper hepatic artery $(P=0.028, \mathrm{HR}=2.089)$ were independent predictors of better OS (Table 2).

\section{Discussion}

The treatment of advanced HCC is still controversial. According to BCLC staging and treatment criteria, systemic therapies are the standard treatment for this stage of HCC, and the reported median OS was 6.5-11.8 months when treated with sorafenib [2]. However, based on the Chinese primary liver cancer diagnosis and treatment guidelines, HCC patients with vascular invasion and/or extrahepatic metastasis are recommended for treatment with TACE, systemic therapy, surgical resection, radiotherapy or combination therapy [3]. Apatinib is a novel vascular endothelial growth factor receptor 2 kinase inhibitor that has been reported to be effective against a variety of solid tumors [13]. In this study, we demonstrated that TACE combined with apatinib achieves a median OS of up to 15 months and that hand-foot syndrome, secondary hypertension and reduction rate of proper hepatic artery were independent predictors of better OS.

Although the results of sorafenib combined with TACE in the treatment of advanced HCC were not satisfactory, the combination of apatinib with TACE may provide a new method of combination therapy. Several retrospective studies compared sorafenib combined with TACE versus sorafenib alone for advanced HCC and found that combination therapy improved the patients' survival $[14,15]$. However, a recent phase III clinical trial showed that the addition of TACE to sorafenib failed to achieve significant survival benefit [6]. The reported median OS in sorafenib plus TACE group ranged between 12.8 and 17.3 months $[6,14-16]$. In contrast, although the combination of apatinib with TACE was compared with TACE alone, the median OS of the combined treatment group was reported to be 11.9-22 months [17-21]. This study showed that the median OS of 51 patients with advanced HCC was 15 months, which is in accordance with the previous reports [9-11]. On the other hand, the clinical study of sorafenib combined with TACE versus sorafenib alone in the treatment of progressive HCC showed that combination therapy can significantly improve tumor response rate and PFS, with reported median PFS of 5.2-5.4 months $[6,16]$. When treated with apatinib combined with TACE, several studies reported median PFS for advanced HCC of 4.5-9.5 months [10,19]. Our results showed a median PFS of up to 10 months. These data seem to suggest that the combination of apatinib with TACE is a promising combination treatment.

There are no established reliable biomarkers to predict the efficacy of systemic therapy with or without TACE for HCC so far. Alternatively, several previous studies evaluated possible factors affecting survival but yielded conflicting results. Yang et al [22] used apatinib combined with TACE to treat advanced HCC and found that patient survival was not associated with any apatinib-related adverse events but with macrovascular invasion and extrahepatic metastases. In contrast, Liao et al [23] treated stage IV sarcoma 
with apatinib and found that post-treatment hypertension, hand-foot syndrome, and proteinuria indicated a favorable prognosis. Our present results supported the findings that apatinib-related adverse events may represent independent predictors for patient survival. These adverse events are probably related to the antiangiogenic effect of apatinib by inhibition of vascular endothelial growth factor receptor 2 .

A surprising finding was that patients with mildly reduced proper hepatic artery diameter after combination therapy had longer OS than those with severely reduced diameter, which seems to contradict the anti-angiogenic effect of apatinib. In a previous phase II trial, Siegel et al. [24] evaluated the efficacy of bevacizumab, an anti-VEGF monoclonal antibody, in unresectable HCC and found that the mean tumor enhancement on contrast-enhanced MR imaging was significantly decreased after treatment, which was positively correlated with reduction in tumor diameter. The present study employed the change of hepatic artery diameter instead of tumor enhancement as the intratumoral necrosis and lipiodol deposition after TACE impaired the results of measuring tumor enhancement on CT. Our results showed that degree of the proper hepatic artery reduction after combination therapy was negatively correlated with OS. This finding is likely related to hepatic arterial damage caused by TACE. It has been reported that TACE can cause hepatic artery stenosis or occlusion [25]. In the present study, patients received 1 or more TACE treatments before initiation of apatinib treatment. The damage of the hepatic artery caused by TACE may interfere with the analysis of hepatic artery size.

\section{Conclusions}

One of the major limitations of this study was that this was a retrospective study, which was inevitably subject to biases affecting such studies. In addition, our study did not include a standard systemic or TACE monotherapy group. Nevertheless, findings from the current study show that TACE combined with apatinib represents an effective combination therapy for advanced HCC, and that hand-foot syndrome, secondary hypertension and the reduction rate of proper hepatic artery were associated with OS. Additional prospective trials with larger sample sizes are warranted to validate our findings.

\section{Declarations}

\section{Funding}

This study was supported by the National Natural Science Foundation of China (No. 81771950, 81471765).

\section{Authors' contributions}

$\mathrm{ZL}, \mathrm{XK}$ : acquisition of data, interpretation of data, statistical analyses, drafting the manuscript. LZ, JM, QL, DZ: acquisition of data, interpretation of data. GZ, GF, CZ: critical revision of the manuscript for important intellectual content, performed transarterial chemoembolization. BL: study concept and design, acquisition of data, interpretation of data, critical revision of the manuscript for important intellectual content, performed transarterial chemoembolization. All authors read and approved the final manuscript. 


\section{Ethics approval and consent to participate}

This retrospective study was approved by the Ethics Committee of the Wuhan Union Hospital, Tongji Medical College, Huazhong university of Science and Technology. Written informed consent was obtained from all patients.

\section{Availability of data and materials}

The data analysed during this study are avaliable from the electrical medical database of Union Hospital, Tongji Medical college, Huazhong University of Science and Technology. Please contact the author Bin Liang (bliang@hust.edu.cn) uponreasonable requests.

\section{Consent for publication}

Not applicable.

\section{Competing interests}

The authors declare that they have no competing interests.

\section{Acknowledgement}

We thank to all the medical workers in our department for their assistance with the study, without their encouragement and assistance this thesis would not have been completed.

\section{Abbreviations}

OS: overall survival; TACE, transarterial chemoembolization; HCC, hepatocellular carcinoma; PFS: progression-free survival; HIF-1, hypoxia-inducible factor-1; VEGF, vascular endothelial growth factor; HBV, hepatitis B virus; ECOG, Eastern Cooperative Oncology Group; AFP, alpha fetoprotein

\section{References}

1. Forner A, Reig M, Bruix J. Hepatocellular carcinoma. Lancet. 2018;391:1301-14.

2. European Association for the Study of the Liver. EASL Clinical Practice Guidelines: Management of hepatocellular carcinoma. J Hepatol. 2018;69:182-236.

3. Zhou J, Sun HC, Wang Z, Cong WM, Wang JH, Zeng MS, et al. Guidelines for Diagnosis and Treatment of Primary Liver Cancer in China (2017 Edition). Liver Cancer. 2018;7:235-60.

4. Gaba RC, Lokken RP, Hickey RM, Lipnik AJ, Lewandowski RJ, Salem R, et al. Quality Improvement Guidelines for Transarterial Chemoembolization and Embolization of Hepatic Malignancy. J Vasc Interv Radiol. 2017;28:1210-23. 
5. Liang B, Zheng CS, Feng GS, Wu HP, Wang Y, Zhao H, et al. Correlation of hypoxia-inducible factor 1alpha with angiogenesis in liver tumors after transcatheter arterial embolization in an animal model. Cardiovasc Intervent Radiol. 2010;33:806-12.

6. Park JW, Kim YJ, Kim DY, Bae SH, Paik SW, Lee YJ, et.al. Sorafenib with or without concurrent transarterial chemoembolization in patients with advanced hepatocellular carcinoma: The phase III STAH trial. J Hepatol. 2019;70:684-91.

7. Ding J, Chen X, Gao Z, Dai X, Li L, Xie C, et.al. Metabolism and pharmacokinetics of novel selective vascular endothelial growth factor receptor-2 inhibitor apatinib in humans. Drug Metab Dispos. 2013;41:1195-210.

8. Lu W, Jin XL, Yang C, Du P, Jiang FQ, Ma JP, et al. Comparison of efficacy between TACE combined with apatinib and TACE alone in the treatment of intermediate and advanced hepatocellular carcinoma: $A$ single-center randomized controlled trial. Cancer Biol Ther. 2017;18:433-8.

9. Chen S, Yu W, Zhang K, Liu W. Comparison of the efficacy and safety of Transarterial chemoembolization with and without Apatinib for the treatment of BCLC stage $C$ hepatocellular carcinoma. BMC Cancer. 2018;19;18:1131.

10. Yang Z, Chen G, Cui Y, Xiao G, Su T, Yu J, et al. The safety and efficacy of TACE combined with apatinib on patients with advanced hepatocellular carcinoma: a retrospective study. Cancer Biol Ther. 2019;20:321-7.

11. Zhu Y, Feng B, Mei L, Sun R, Guo C, Zhu J. Clinical efficacy of TACE combined with Apatinib in the treatment of advanced hepatocellular carcinoma. J BUON. 2019;24:608-14.

12. Fan W, Yuan G, Fan H, Li F, Wu Y, Zhao Y, et al. Apatinib combined with transarterial chemoembolization in patients with hepatocellular carcinoma and portal vein tumor thrombus: a multicenter retrospective study. Clin Ther. 2019;41:1463-76.

13. Scott LJ. Apatinib: A Review in Advanced Gastric Cancer and Other Advanced Cancers. Drugs. 2018;78:747-58.

14. Kok VC, Chen YC, Chen YY, et.al. Yoshida GJ. Sorafenib with Transarterial Chemoembolization Achieves Improved Survival vs. Sorafenib Alone in Advanced Hepatocellular Carcinoma: A Nationwide Population-Based Cohort Study. Cancers (Basel). 2019;11.pii: E985.

15. Chien SC, Chen CY, Cheng PN, Liu YS, Cheng HC, Chuang CH, et.al. Combined Transarterial Embolization/Chemoembolization-Based Locoregional Treatment with Sorafenib Prolongs the Survival in Patients with Advanced Hepatocellular Carcinoma and Preserved Liver Function: A Propensity Score Matching Study. Liver Cancer. 2019;8:186-202.

16. Sato Y, Nishiofuku H, Yasumoto T, Nakatsuka A, Matsuo K, Kodama Y, et al. Multicenter Phase II Clinical Trial of Sorafenib Combined with Transarterial Chemoembolization for Advanced Stage Hepatocellular Carcinomas (Barcelona Clinic Liver Cancer Stage C): STAB Study. J Vasc Interv Radiol. 2018;29:1061-7.

17. Ni JY, Kong J, Sun HL, Chen YT, Luo JH, Wang WD, et.al. Prognostic Factors for Survival After Transarterial Chemoembolization Combined with Sorafenib in the Treatment of BCLC Stage B and C Hepatocellular Carcinomas. Acad Radiol. 2018;25:423-9.

18. Yang Z, Chen G, Cui Y, Xiao G, Su T, Yu J, et.al. The safety and efficacy of TACE combined with 
apatinib on patients with advanced hepatocellular carcinoma: a retrospective study. Cancer Biol Ther. 2019;20:321-7.

19. Liu C, Xing W, Si T, Yu H, Guo Z. Efficacy and safety of apatinib combined with transarterial chemoembolization for hepatocellular carcinoma with portal venous tumor thrombus: a retrospective study. Oncotarget. 2017;8:100734-5.

20. Liu J, Xu J, Zhang W, Chen J, Zhou X, Li Z, et.al. Safety and Efficacy of Drug-Eluting Bead Transarterial Chemoembolization Combined with Apatinib in Patients with Advanced Hepatocellular Carcinoma. Acad Radiol. 2019;pii:S1076-6332(19)30333-2.

21. Fan W, Yuan G, Fan H, Li F, Wu Y, Zhao Y, et.al. Apatinib Combined With Transarterial Chemoembolization in Patients With Hepatocellular Carcinoma and Portal Vein Tumor Thrombus: A Multicenter Retrospective Study. Clin Ther. 2019;41:1463-76.

22. Yang Z, Chen G, Cui Y, Xiao G, Su T, Yu J, et.al. The safety and efficacy of TACE combined with apatinib on patients with advanced hepatocellular carcinoma: a retrospective study. Cancer Biol Ther. 2019;20:321-7.

23. Liao Z, Li F, Zhang C, Zhu L, Shi Y, Zhao G, et.al. Phase II trial of VEGFR2 inhibitor apatinib for metastatic sarcoma: focus on efficacy and safety. Exp Mol Med. 2019;51:24.

24. Siegel AB, Cohen El, Ocean A, Lehrer D, Goldenberg A, Knox JJ, et.al. Phase II trial evaluating the clinical and biologic effects of bevacizumab in unresectable hepatocellular carcinoma. $\mathrm{J}$ Clin Oncol. 2008;26:2992-8.

25. Lee S, Kim KM, Lee SJ, Lee KH, Lee DY, Kim MD, et.al. Hepatic arterial damage after transarterial chemoembolization for the treatment of hepatocellular carcinoma: comparison of drug-eluting bead and conventional chemoembolization in a retrospective controlled study. Acta Radiol. 2017;58:131-9.

\section{Tables}

Table 1 Patient characteristics and univariate analysis for overall survival 


\begin{tabular}{|c|c|c|c|}
\hline Variable & Number of patients & $\begin{array}{l}\text { Median OS } \\
\text { (month) }\end{array}$ & $P$ value \\
\hline \multicolumn{4}{|l|}{ HBV } \\
\hline Negative & $4(7.8 \%)$ & 10 & 0.447 \\
\hline Positive & $47(92.2 \%)$ & 15 & \\
\hline \multicolumn{4}{|l|}{ ECOG score } \\
\hline 0 & $0(0)$ & & 0.373 \\
\hline 1 & $42(82.4 \%)$ & 15 & \\
\hline 2 & $9(17.6 \%)$ & 15 & \\
\hline \multicolumn{2}{|l|}{ Child-Pugh grade } & & 0.008 \\
\hline A & $44(86.3 \%)$ & 17 & \\
\hline B & $7(13.7 \%)$ & 10 & \\
\hline AFP & & & 0.076 \\
\hline$<400 \mathrm{ug} / \mathrm{L}$ & $23(45.1 \%)$ & 19 & \\
\hline$\geq 400 \mathrm{ug} / \mathrm{L}$ & $28(54.9 \%)$ & 12 & \\
\hline \multicolumn{2}{|l|}{ Number of lesions } & & 0.666 \\
\hline 1 & $17(33.3 \%)$ & 15 & \\
\hline$\geq 2$ & $34(66.7 \% \square$ & 15 & \\
\hline \multicolumn{2}{|l|}{ Size of dominant lesion } & & 0.647 \\
\hline$<5 \mathrm{~cm}$ & $12(23.5 \%)$ & 18 & \\
\hline $5-10 \mathrm{~cm}$ & $18(35.3 \%)$ & 17 & \\
\hline$>10 \mathrm{~cm}$ & $21(41.2 \%)$ & 14 & \\
\hline \multicolumn{2}{|l|}{ Macrovascular invasion } & & 0.363 \\
\hline None & $26(51.0 \%)$ & 15 & \\
\hline Portal vein & $22(43.1 \%)$ & 13 & \\
\hline $\begin{array}{l}\text { HV and/or IVC } \\
\text { inferior vena cava }\end{array}$ & $3(5.9 \%)$ & 19 & \\
\hline \multicolumn{2}{|l|}{ Extrahepatic spread } & & 0.285 \\
\hline None & $18(35.3 \%)$ & 14 & \\
\hline Lymph node metastasis & $11(21.6 \%)$ & 15 & \\
\hline Peritoneal metastasis & $9(17.6 \%)$ & 18 & \\
\hline Organ metastasis & $13(25.5 \%)$ & 12 & \\
\hline \multicolumn{2}{|l|}{ Ascites } & & 0.013 \\
\hline Present & $8(15.7 \%)$ & 9 & \\
\hline Absent & $43(84.3 \%)$ & 17 & \\
\hline \multicolumn{2}{|l|}{ Number of TACE } & & 0.227 \\
\hline 1 & $32(62.7 \%)$ & 13 & \\
\hline$\geq 2$ & $19(37.3 \%)$ & 15 & \\
\hline \multicolumn{2}{|l|}{ The proper hepatic artery } & & 0.013 \\
\hline Mild reduction & $25(49.0 \%)$ & 23 & \\
\hline Severe reduction & $26(51.0 \%)$ & 12 & \\
\hline \multicolumn{2}{|l|}{ Hand-foot syndrome } & & 0.006 \\
\hline None & $13(25.5 \%)$ & 10 & \\
\hline Grade 1 & $11(21.6 \%)$ & 14 & \\
\hline Grade 2 & $24(47.1 \%)$ & 21 & \\
\hline Grade 3 & $3(5.9 \%)$ & 17 & \\
\hline \multicolumn{2}{|l|}{ Secondary hypertension } & & 0.042 \\
\hline None & $22(43.1 \%)$ & 15 & \\
\hline Grade 1 & $18(35.3 \%)$ & 18 & \\
\hline Grade 2 & $9(17.6 \%)$ & 16 & \\
\hline Grade 3 & $2(3.9 \%)$ & 28 & \\
\hline \multicolumn{2}{|l|}{ Diarrhea } & & 0.102 \\
\hline None & $40(78.4 \%)$ & 14 & \\
\hline Grade 1 & 7 (13.7\%) & 18 & \\
\hline Grade 2 & $4(7.8 \%)$ & 15 & \\
\hline \multicolumn{4}{|l|}{ Proteinuria } \\
\hline None & 29 (56.9\%) & 14 & 0.373 \\
\hline Grade 1 & $6(11.8 \%)$ & 15 & \\
\hline Grade 2 & $16(31.3 \%)$ & 19 & \\
\hline
\end{tabular}

Abbreviations: HBV, hepatitis B virus; ECOG, Eastern Cooperative Oncology Group; AFP, alpha fetoprotein; HV, hepatic vein; IVC, inferior vena cava; TACE, transarterial chemoembolization; OS, overall survival. 
Table 2 Multivariate analysis for overall survival

\begin{tabular}{llll}
\hline Parameter & Hazard ratio & $95 \%$ CI & $P$ value \\
\hline Child-pugh grade & 0.955 & $0.353-2.582$ & 0.928 \\
AFP & 1.935 & $0.925-4.049$ & 0.080 \\
\hline Ascites & 1.934 & $0.776-4.823$ & 0.157 \\
\hline The proper hepatic artery & 2.089 & $1.081-4.038$ & 0.028 \\
\hline Hand-foot syndrome & 0.554 & $0.369-0.830$ & 0.004 \\
\hline Secondary hypertension & 0.647 & $0.432-0.967$ & 0.034 \\
\hline
\end{tabular}

Abbreviations: AFP, alpha fetoprotein.

Figures

Outline criteria: $O$ diffuse $\mathrm{HCC}$ or liver tumor burden $>50 \%$ of total liver volume $O$ complete obstruction of the main portal vein, advanced liver disease $O$ severe dysfunction of kidney, heart, lung or coagulation.

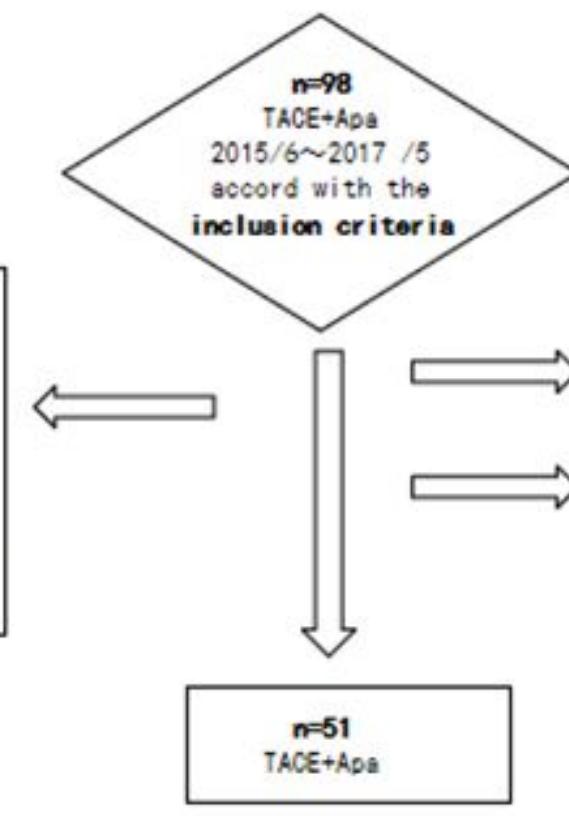

$\mathrm{n}=\mathbf{1 8}$ discontinued apatinib $>1$ month or was lost to follow-up

$\mathbf{n}=\mathbf{2 9}$ lose to follow-up

\section{Figure 1}

Flow chart of patients who were included in this study. 

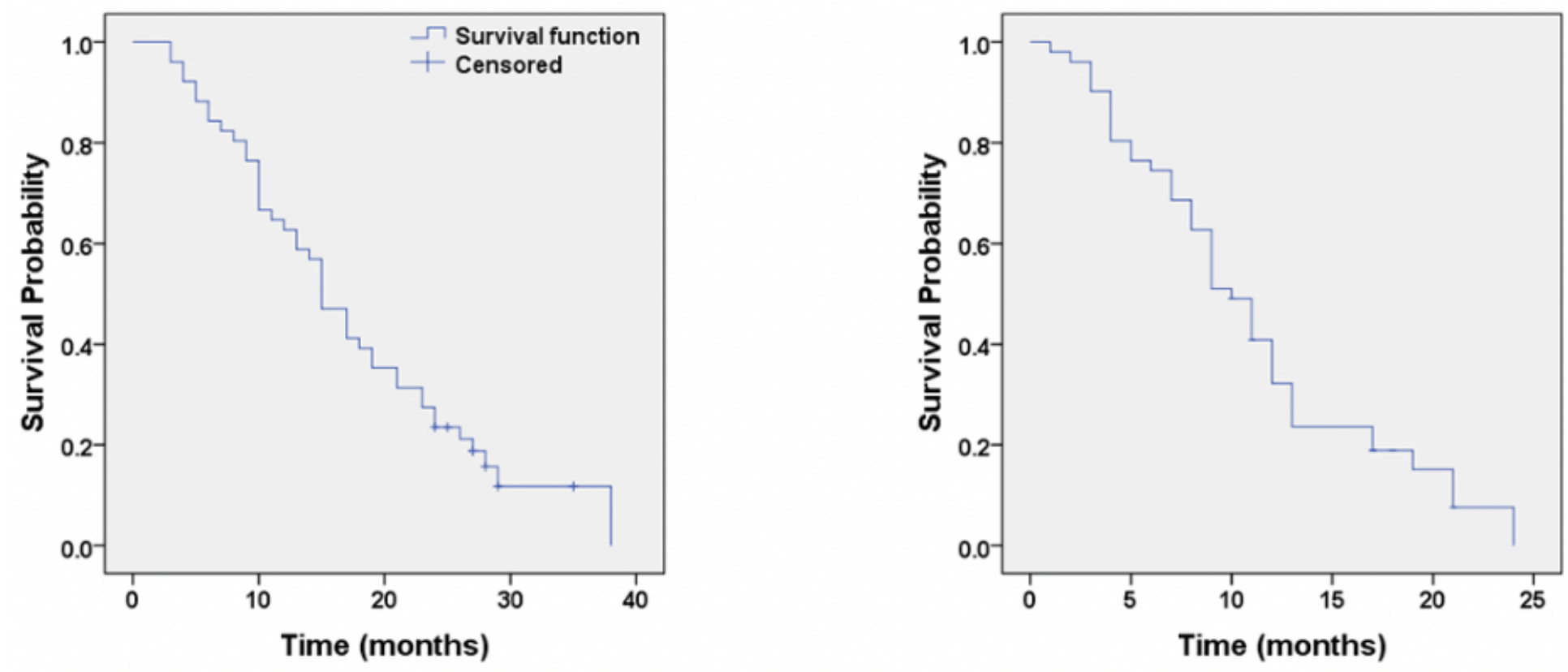

\section{Figure 2}

The median follow-up time was 15 months (range, 3-38 months). Forty-four (86.3\%) of the 51 patients died and 7 (13.7\%) remained alive during the follow-up period (June 2015 to May 2019). The median OS was 15 months (range, 3-38 months) (Fig. 2a), and the median PFS was 10 months (range, 1-24 months) (Fig. 2b). The cumulative OS rates at 1, 2, and 3 years were $62.7 \%, 23.5 \%$, and $1.8 \%$, respectively.
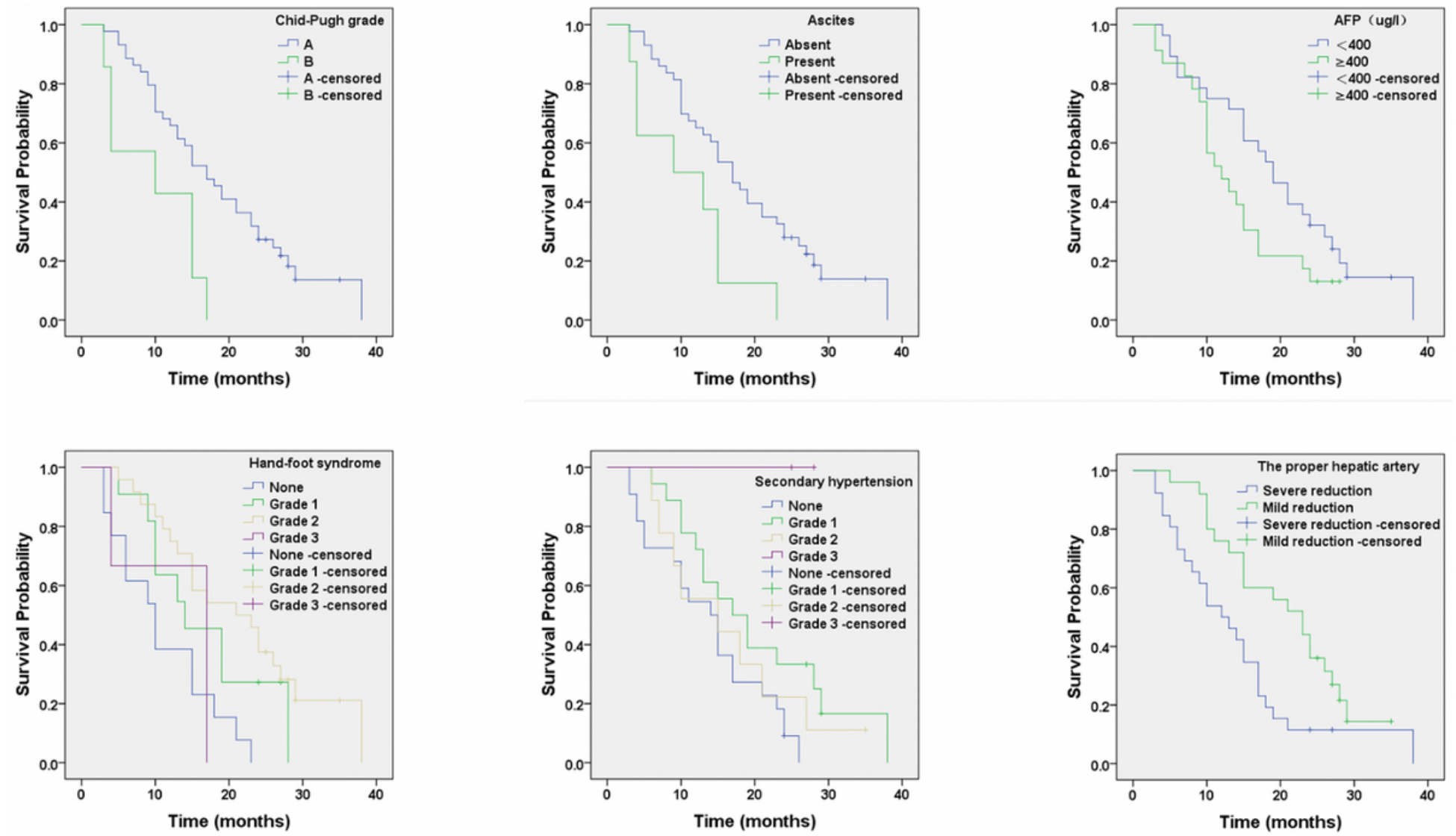

Figure 3 
At univariate analysis, patients with Child-Pugh grade A had significantly longer median OS compared with Child-Pugh grade B patients (17 months vs.10 months, $P=0.008)$ (Fig. 3a). Patients without ascites had significantly longer median OS compared with those with ascites ( 17 months vs. 9 months, $P=$ 0.013) (Fig. 3b). patients with AFP $<400 \mathrm{ug} / \mathrm{L}$ had significantly longer median OS compared with AFP $\geq$ $400 \mathrm{ug} / \mathrm{L}$ patients (19 months vs.12 months, $\mathrm{P}=0.076)(\mathrm{Fig} .3 \mathrm{c}$ ). The median OS of patients without hand-foot syndrome was 10 months, whereas the median OS of patients with grade 1, 2 and 3 hand-foot syndrome was 14, 21 and 17 months, respectively. Patients with grade 2 hand-foot syndrome had significantly longer median OS compared with patients without hand-foot syndrome ( 21 months vs. 10 months, $P=0.003$ ) (Fig. 3d). The median OS of patients without secondary hypertension was 15 months, whereas that of patients with grade 1, 2 and 3 secondary hypertension was 18, 15.5 and 28 months, respectively. Patients with grade 3 hypertension had significantly longer median OS compared with nonhypertensive patients (28 months vs. 15 months, $P=0.032$ ) (Fig. 3e). The diameter of the proper hepatic artery before and after combination therapy was $3.79 \pm 1.81 \mathrm{~mm}$ and $3.40 \pm 1.66 \mathrm{~mm}$, respectively, with a statistically significant difference $(P<0.001)$. The median reduction rate of the proper hepatic artery after treatment was $7.7 \%$. Patients with mildly reduced diameter of the proper hepatic artery had longer median OS than those with severely reduced vessel diameters (23 months vs. 12 months, $P=0.013$ ) (Fig. $3 f$ ) 